\title{
Stability in Cosmology, from Einstein to Inflation
}

\author{
C.D. McCoy
}

\begin{abstract}
I investigate the role of stability in cosmology through two episodes from the recent history of cosmology: (1) Einstein's static universe and Eddington's demonstration of its instability, and (2) the flatness problem of the hot big bang model and its claimed solution by inflationary theory. These episodes illustrate differing reactions to instability in cosmological models, both positive ones and negative ones. To provide some context to these reactions, I also situate them in relation to perspectives on stability from dynamical systems theory and its epistemology. This reveals, for example, an insistence on stability as an extreme position in relation to the spectrum of physical systems which exhibit degrees of stability and fragility, one which has a pragmatic rationale, but not any deeper one.
\end{abstract}

\section{Introduction}

Although the meeting in Bern where this paper was presented commemorated Einstein's completion of his general theory of relativity, its date marks the 100th anniversary of the publication of another of his famous papers, "Kosmologische Betrachtungen zur allgemeinen Relativitätstheorie" (Cosmological Considerations in the General Theory of Relativity) (Einstein, 1917), the founding paper of the field of relativistic cosmology. In this paper Einstein proposed an unchanging, temporally infinite, and spatially finite relativistic model of the large-scale universe, the so-called 'Einstein static universe'. Other cosmological models of general relativity soon followed, for example by de Sitter (1917a,b,c), Friedman (1922, 1924), and Lemaître (1927). Despite mounting observational evidence that the universe was not static and was in fact expanding, as these latter models allowed, Einstein maintained belief in his model for many years, until finally publicly disavowing it in (Einstein,

C.D. McCoy

School of Philosophy, Psychology, and Language Sciences, University of Edinburgh, Dugald Stewart Building, 3 Charles Street, Edinburgh, EH8 9AD, UK, e-mail: casey.mccoy@ed.ac.uk 
1931). The decisive point for Einstein was not this observational evidence however; rather he seems to have been convinced by Eddington of the unsuitability of his model due to its instability (Nussbaumer, 2014).

So began a minor, albeit sometimes significant thread through the history of modern cosmology, tying together episodes involving the theme of stability. It is this theme which is my topic. While there are, admittedly, many greater themes in the history of cosmology, stability has nevertheless on occasion played an appreciable and important role, as I aim to show. So, what has been the role of stability considerations in cosmology? And also, has the attitude to stability changed over the years? This latter question is especially worth considering, when we remind ourselves of the great advances in the study of nonlinear dynamics which crucially involve the concept of instability, such as the development of chaos theory. To help answer these questions I examine the Einstein-Eddington episode just mentioned (\$2), and also the more recent 'flatness problem' of the big bang model (\$3), which ushered in the contemporary paradigm of inflationary cosmology in the 1980s.

As said, Einstein himself was ultimately convinced to abandon his cosmological model on stability grounds, when nearly all other physicists had already been suspicious of it on observational grounds well before 1931. Whereas Einstein cited the instability of the model as cause enough for rejecting his model, other physicists, such as Eddington and Lemaitre, instead sought to make use of this instability as a means for effecting a transition to an expanding universe, through some manner of physical perturbation. Although the static universe idea maintained some currency in the following decades, it was eventually dropped altogether by cosmologists in favor of the past-finite expanding universe models of Friedman and Lemaître, in particular in the guise of the hot big bang model, developed especially by Gamow and his collaborators in the 1940s.

Later, however, the big bang model was brought into question due to its alleged 'fine-tuning problems', which led to the widespread adoption of inflationary theory in the 1980s on the grounds (at least initially) that it solved these problems. One of these problems is the flatness problem. The flatness problem begins with observations which have increasingly suggested that the spatial geometry of the universe is nearly flat (or Euclidean). The spatially flat big bang models are dynamically unstable however: any slight deviation from flatness results in an increasingly divergent curvature. Yet this instability was not given by cosmologists as the sole reason to seek an alternative model; rather, as a consequence of this instability, they inferred from the observed high degree of flatness an extraordinary degree of fine-tuning in the initial conditions of the universe. It is this fine-tuning that was taken by cosmologists as the reason to reject the simple big bang model and implement a dynamical means (inflation) for insuring, among other things, the stability of spatial flatness.

Even this quick sketch evinces an interesting variety of attitudes towards stability held by the physicists involved. Nevertheless, when one scrutinizes their arguments, it is not readily apparent why they took the attitudes that they did. It is not clear, for example, what they took the physical significance of instability (or lack thereof) to be, how to characterize the physical source of the perturbations they imagine, which variations matter, how to ground the 'improbability' of finely-tuned states, 
and so on. Greater historical and individual context are surely needed to answer these questions fully. However, some useful progress can be made by considering a seemingly once common conceptual paradigm: that stability is a necessary feature of physical systems (and models based on them). The ubiquity of this paradigm, perhaps, can account for some reactions to the instability of the Einstein static universe. Later, this paradigm came to be seen as mere dogma thanks to the aforementioned advances in dynamical systems theory, as it became ever more evident that one should not, indeed cannot, expect dynamical models to be stable, except in very simple cases. Models exhibiting aspects of what is sometimes called fragility, that is, some degree of instability, have found important applications in physics and beyond, a particularly notable example, again, being those models studied in chaos theory. Many ideas and much mathematics concerning such models were developed even before the 20th century, yet the widespread realization of their physical significance and ubiquity has grown out of research in nonlinear dynamical systems only since the 1960s. In the final section of the paper (§4), I draw especially on ideas and arguments presented in (Tavakol, 1991) concerning these developments in order to assess and reflect on the theme of stability in cosmology, particularly the two episodes discussed in the next two sections.

\section{The Einstein Static Universe}

The first episode is the story of Einstein's static universe model. Besides introducing this model, the first relativistic spacetime model of the universe, (Einstein, 1917) is also famous for his modification of the field equations to include the cosmological constant. ${ }^{1} \mathrm{He}$ was moved to make this modification in light of what he took to be a certain paradox in cosmological models involving gravitation, both Newtonian and relativistic. This paradox is not so relevant to the discussion here, nor are most of Einstein's motivations for introducing his cosmological model and the cosmological constant. $^{2}$ Thus, rather than following Einstein's fairly idiosyncratic presentation of his model, I will specify the Einstein static universe in modern geometrical terms, for this will help make the structure of the model and its instability more manifest.

In these terms one understands a relativistic spacetime as a geometrical model of the general theory of relativity (Hawking and Ellis, 1973; Wald, 1984; Malament, 2012). It is a differentiable manifold $\mathcal{M}$ equipped with a Lorentzian metric field $g$. The matter content of the spacetime is specified by the stress energy tensor field $T$ and the cosmological constant is denoted by the scalar field $\Lambda$. The metric field $g$ and stress energy field $T$ associated with $\mathcal{M}$ satisfy the Einstein field equations with cosmological constant (EFE- $\Lambda$ ):

$$
R-\frac{1}{2} \mathcal{R} g-\Lambda g=8 \pi T,
$$

\footnotetext{
${ }^{1}$ For commentaries on this paper, see (Smeenk, 2014; O'Raifeartaigh et al, 2017).

${ }^{2}$ See, e.g., (Norton, 1992; Earman, 2001; Kragh, 2007) for discussions of this background.
} 
where $R$ is the Ricci tensor and $\mathcal{R}$ is the Ricci scalar, both obtainable from $g$. The Einstein static universe can then be specified as the non-vacuum $(T \neq 0)$ relativistic spacetime which satisfies the following conditions: (1) it is spatially homogeneous and isotropic, and (2) it is static.

A spacetime that is spatially homogeneous and isotropic possesses a congruence of timelike curves such that it is possible to foliate spacetime by a one-parameter family of spacelike hypersurfaces orthogonal to the timelike curves. Given this foliation, spatial homogeneity and isotropy jointly require that there exists some timelike vector field such that for each spatial hypersurface $\Sigma_{\lambda}$, where $\lambda$ denotes the temporal parameter along the timelike curves, the geometrical characteristics of all points in each such hypersurface are the same. ${ }^{3}$ With these symmetries, the matter content of the spacetime can be represented as a perfect fluid with energy density $\rho$ and pressure $p$. The EFE- $\Lambda$ will then reduce to two coupled equations (the Friedman equations):

$$
\begin{gathered}
3 H^{2}+3 \kappa=8 \pi \rho+\Lambda ; \\
3 \dot{H}+3 H^{2}+4 \pi(\rho+3 p)=\Lambda,
\end{gathered}
$$

where $H$ is the Hubble parameter, specifying the expansion of space, and $\kappa$ is the curvature of space. ${ }^{4}$ Only $\Lambda$ is assumed to remain constant in time (also, derivatives with respect to the temporal parameter are denoted by overdots). The spacetimes thus obtained are generally called the Friedman(-Lemaître)-Robertson-Walker (FRW or FLRW) models.

Among the FRW models the Einstein static universe is a special spacetime due to the second condition: staticity. A static FRW spacetime is one where $H=0$; that is, it neither expands nor contracts. This simplifies the EFE- $\Lambda$ equations further, to

$$
\begin{gathered}
3 \kappa=8 \pi \rho+\Lambda \\
4 \pi(\rho+3 p)=\Lambda .
\end{gathered}
$$

Einstein's paper considers only the case where matter is pressureless 'dust'. In this special case $(p=0)$, the universe is static when $\Lambda=4 \pi \rho=\kappa \cdot{ }^{5}$ It also follows that space is positively curved in the static universe, since $\kappa=4 \pi \rho>0$, and that $\Lambda$ is a positive cosmological constant. For if $\kappa=0$, then $\rho=\Lambda=0$-we have Minkowski spacetime, a spacetime without matter, and if $\kappa<0$, then $\rho<0$, violating the weak energy condition. Thus, the only non-vacuum spacetime that satisfies the Einstein static universe conditions is the positively curved one. Since the only possible spatial

\footnotetext{
${ }^{3}$ A more precise characterization of this condition are given in (Malament, 2012, §2.11). See also (McCabe, 2004; McCoy, 2016).

${ }^{4}$ The Hubble parameter $H$ is related to the expansion scalar $\theta$ (from the Raychaudhuri equation) by $\theta=3 H$. The spatial curvature $\kappa$ is related to the Ricci scalar $\mathcal{R}_{\Sigma}$ of the spatial hypersurfaces in FRW spacetimes by $\mathcal{R}_{\Sigma}=-6 \kappa$. Cf. (Malament, 2012, §2.11). Note that my $\kappa$ is the negative of Malament's $\mathcal{K}$ and he uses normal and script fonts to differentiate the spacetime and spatial Ricci tensors and scalars, respectively, where I introduce subscripts for the the spatial ones.

${ }^{5}$ Cf. (Einstein, 1923, 187). See also (Malament, 2012, 194).
} 
topology of such a spacetime is the sphere, it follows that the Einstein static universe is spatially finite, a circumstance which Einstein found particularly favorable. ${ }^{6}$

In the more general case, where $p \neq 0$, we may repeat the previous argument, obtaining essentially the same results. If $\kappa=0$, then $\rho=-p$ and the 'matter' content of the universe acts as an inverse cosmological constant which offsets the 'actual' cosmological constant $\Lambda$. In other words, we have just found Minkowski spacetime again. If $\kappa<0$, then $\rho<0$ or $\rho+p<0$, in either case violating the weak energy condition. Thus, only in the case that $\kappa>0$ do we have a physically reasonable non-vacuum spacetime, and, again, it is one that is spatially finite.

In the same year as Einstein proposed the static universe model, de Sitter proposed an alternative model of the universe (de Sitter, 1917a,b,c). For the sake of comparison, it is worth describing his model briefly. The de Sitter universe is a vacuum spacetime (at large scales, anyway) which expands at a constant rate due to the presence of, in effect, a (positive) cosmological constant. Assuming spatial homoegeneity and isotropy again for the sake of comparison to FRW models, the EFE- $\Lambda$ in this case reduce to

$$
\begin{aligned}
& 3 H^{2}+3 \kappa=\Lambda \\
& 3 \dot{H}+3 H^{2}=\Lambda .
\end{aligned}
$$

If we choose a foliation of de Sitter spacetime where the spatial hypersurfaces are flat $(\kappa=0)$, we see that $\dot{H}=0$ and

$$
3 H^{2}=\Lambda
$$

that is, spatial expansion (given our arbitrary choice of what space is in the de Sitter universe) is constant and proportional to the (positive) cosmological constant. As a consequence, geodesics diverge from one another exponentially in time. Also, like the Einstein static universe, time in the de Sitter universe is past and future infinite, so the the de Sitter universe neither begins nor ends.

Much of the debate in the 1920s focused on these two cosmological models. Despite the lack of matter in de Sitter's universe, it made a stronger connection to available cosmological observations, especially by furnishing a possible explanation for Slipher's recent observations of redshifting in the spectral lines of galaxies (Slipher, 1912, 1915, 1917, 1921). Einstein was unaware of the latest observational results in astronomy and instead relied on his intuition about the nature of the universe, holding 'philosophically' that it was static and spatially finite. Uncertainty and confusion about coordinate choices and the geometrical structure of the models, as well as about the actual physical features of the universe accessible through observation, stymied understanding for many years. Perhaps for these reasons, it

${ }^{6}$ Thus, $\mathcal{M}=S^{3} \times \mathbf{R}$, which is why the Einstein static universe is sometimes called the "cylindrical universe'. 
was not until end of the decade that the instability of the Einstein static universe was noticed and communicated by Eddington (1930). ${ }^{7}$

Eddington considers only a small scalar perturbation of the density $\rho$ in a universe consisting of dust $(p=0)$. Combining the Friedman equations by eliminating the curvature term, we have

$$
\dot{H}=\Lambda-4 \pi \rho .
$$

As noted above, the Einstein static universe is the universe where $4 \pi \rho=\Lambda$. Clearly a "slight disturbance" which causes $\rho$ to increase or decrease will lead the universe to contract or expand, respectively - "evidently Einstein's universe is unstable" (Eddington, 1930, 670). We might also consider the case where the pressure is positive, in which case we have

$$
\dot{H}=\Lambda-4 \pi(\rho+p) .
$$

A slight disturbance in the density or the pressure would, however, again clearly lead to a changing Hubble parameter.

One might easily question Eddington's demonstration on a couple of points. First, why should the relevant equations of motion be those pertaining to the FRW class of spacetimes and not all relativistic spacetimes? Eddington considers only a single kind of perturbation, one which is consistent with such universes: a homeogeneous and isotropic spacelike perturbation of the energy density. This is enough to show that the Einstein static universe is unstable, if we suppose that a model is unstable when it is unstable with respect to any perturbation. Still, one might wonder whether such a perturbation is physically significant. Eddington and other cosmologists thought so for a time, adopting a vision of the universe as having been in a past-infinite state described by Einstein's model, but which at some point transitions via a perturbation to an expanding epoch (and eventually becoming approximated by de Sitter's model after much expansion) (Robertson, 1933). What physical process could lead to a perturbation of this kind in the Einstein static universe?

In typical physical systems, a model that is dynamically unstable is liable to be pushed out of the unstable state by physical perturbations from the environment. Indeed, one might even say that it is improbable for a system to persist in such a state for any appreciable amount of time. But this simplistic idea cuts no ice in cosmology, as there is no external environment from which perturbations impinge on the universe. Obviously the perturbation could not come from 'outside the universe'. Such a perturbation would be "supernatural", as Eddington says.

He claims, however, that "the initial small disturbance can happen without supernatural interference" (Eddington, 1930, 670). The proposal he initially moots is that the gravitational collapse of "uniformly diffused nebula" into galaxies would lead to just such a perturbation: "the actual mass may not alter but the equivalent mass to be used in applying the equations for a strictly uniform distribution must be slightly altered" (Eddington, 1930, 670). But if no new mass would be created in gravitational collapse, it is difficult to see why the 'equivalent mass' used in the

7 Eddington acknowledges Lemaître's investigation into the Einstein and de Sitter universes (Lemaître, 1927), where, he says, "it is at once apparent from his formulæ that the Einstein world is unstable" (Eddington, 1930, 668). 
Friedman equations would change. If the volume of space does not change, and the amount of matter in space does not change, how could the energy density change? This could occur under some conversion process of matter into radiation, which, as Eddington notes, would not change $\rho$ but would change $p$. If such a conversion were to occur, though, it ought to lead to an increase in $\rho$ or $p$, which would lead to spatial contraction. ${ }^{8}$ As the aforementioned redshift observations by Slipher already suggested, along with Hubble's estimates of the distances to galaxies (Hubble, 1929), space should be expanding in the model, not contracting. Thus a physical mechanism which could result in the Einstein static universe falling out of its static 'equilibrium' into an expanding universe seems precluded.

Nevertheless, Eddington's argument that the static universe was unstable convinced Einstein to abandon his model, as the latter acknowledged in (Einstein, 1931). That paper is perhaps better known as the paper in which he abandons the "unsatisfactory" cosmological constant, instead favoring the spherical, expanding FRW spacetime discovered first by Friedman (1922). ${ }^{9}$ In the paper Einstein states two principal reasons for abandoning his static universe: (1) that it was unstable, as Eddington had convinced him previously in conversation, and (2) due to the observational results of Hubble. ${ }^{10}$ However, although he acknowledges the observational results indicating the expansion of the universe, he insists that the instability of the model was reason enough not to ascribe physical significance to the static universe.

Now, one might ignore the evident significance of instability to the participants in this episode, noting that the outcome was nothing more than the adoption of models that were more empirically adequate than the Einstein static universe (and the de Sitter universe)—a humdrum example of empirical progress guiding theoretical progress. This is not how the participants reasoned however. Indeed, it is especially notable that their reactions differed to the instability discovered by Eddington. Einstein, for example, saw instability as undermining the physical significance of a model, whereas Eddington, Lemaitre, and others saw it as an opportunity to introduce a mechanism for creating complex structure in the universe.

Unfortunately, none of them expand on their particular views on stability in any of their contributions. One argument, which might be gleaned from various remarks in these papers, begins with the obvious: the universe is not perfectly uniform, as is supposed in the Einstein static universe and the other prominent cosmological models. Now, if we represent deviations from uniformity as perturbations in our models and suppose that the actual universe should be a 'perturbation away' from our favored idealized model, then the perturbed model which represents the actual

\footnotetext{
${ }^{8}$ This point was investigated soon after Eddington's discovery, especially by McVittie and McCrea. See (McCrea and McVittie, 1930, 1931; McVittie, 1931; Dingle, 1933; Tolman, 1934; Sen, 1935a,b). Lemaittre proposed an alternative mechanism for the departure from equilibrium, which he called a "stagnation" (Lemaître, 1931).

${ }^{9}$ One year later, Einstein and de Sitter (1932) argued for the flat, expanding FRW spacetime, on the grounds that there is no direct observational evidence for non-zero spatial curvature. This model, the Einstein-de Sitter universe, became the standard model of cosmology for much of the 20th century and will reappear in the following section.

${ }^{10}$ See (O'Raifeartaigh and McCann, 2014) for a translation and discussion of this paper. See also (Nussbaumer, 2014) for further analysis of Einstein's reasons.
} 
universe should not deviate significantly from the static model (or other idealized model) over time. Otherwise the static model is a poor approximation of the actual universe and inapt for prediction, explanation, etc. The instability of the Einstein static universe suggests just this: any slight deviation from the Einstein static universe results in significant qualitative differences between the perturbed model and the unperturbed model.

This objection, however, is not by itself sufficient to reject the static model completely. So long as the static model is a sufficiently good approximation to the actual universe, it can be used to describe and make predictions about the universe. Of course, as it happens the static model fits poorly with observations, which is surely reason enough to reject it. Yet if it had turned out that observations fit it well enough, what reason could instability provide to abandon it, at least as an approximation? It appears that rejecting the Einstein static universe merely on the grounds that it is unstable can only involve a dubious insistence on stability in physical models. In contrast to this attitude, Eddington and other cosmologists held on to the static universe for a time, even despite a lack of any observational evidence for it (and presumably for 'philosophical' reasons), recognizing that its instability could be used to effect a 'phase transition' in the large-scale universe. Thus we see in this episode both a degree of positivization of instabilities (Schmidt, 2011, 223) in cosmology and what appears to be a thorough rejection of them in line with a 'dogma of stability' (Abraham and Marsden, 1978, xxii).

\section{The Flatness Problem}

The second episode is the story of the flatness problem. It is one of the fine-tuning problems of the hot big bang model which led to the widespread adoption of inflationary theory by theoretical cosmologists in the 1980s, due to inflationary theory's alleged solution of them. As mentioned above, the flatness problem begins with observations suggesting that the universe's spatial curvature is approximately flat, when the universe is modeled with a FRW spacetime. The flatness problem arises in part because the Friedman equations can be used to demonstrate the dynamic instability of flat curvature under small perturbations, much in the same way that Eddington showed that the Einstein static universe was unstable. The problem is not just the presence of this instability however. The crucial problem, according to proponents of inflation, is that the initial conditions of the universe had to be extremely fine-tuned, due to this instability, for the universe to be anywhere near as flat as observations suggested. It is this fine-tuning that they reject, not the instability per se. Inflationary theory purports to solve this fine-tuning problem with a short stage of exponential expansion in the very early universe (inflation), which reverses the dynamical stability of FRW universes, thereby making flat FRW spacetimes dynamically stable under perturbations (at least during inflation).

The big bang model of the universe is based on the expanding FRW spacetimes, which were introduced in the previous section. These may have positive, negative, or 
flat spatial curvature, which is determined by the density and pressure of spacetime's contents. It has been an empirical matter to determine what the (large-scale) curvature of space is. Although observations have long suggested that the flat model (the so-called 'Einstein-de Sitter universe' of (Einstein and de Sitter, 1932)) is roughly accurate, it cannot be excluded that space has a positive or negative curvature (nor could it, since observations do not have infinite accuracy). Indeed, for aesthetic reasons, many physicists have preferred the spatially finite, positively curved model (the 'Einstein-Friedman universe' of (Friedman, 1922) and (Einstein, 1931)). In any case, it is this observed approximate flatness that is the empirical fact on which the flatness problem is based.

To see how the problem arises, let us revisit the basic assumptions which lead to the FRW spacetimes. First, we suppose that there is a congruence of timelike curves, such that spacetime can be foliated by a one-parameter family of spacelike hypersurfaces ('Weyl's principle'). Then we assume that there exists a foliation where the spacelike hypersurfaces are homogeneous and isotropic ('the cosmological principle'). The spacetimes satisfying these conditions are the FRW spacetimes. They obey the Friedman equations:

$$
\begin{gathered}
3 H^{2}+3 \frac{k}{a^{2}}=8 \pi \rho ; \\
3 \dot{H}+3 H^{2}+4 \pi(\rho+3 p)=0,
\end{gathered}
$$

where I have replaced the curvature $\kappa$ used above by $k / a^{2}$. The parameter $k$ specifies the sign of the curvature: +1 for positively curved, -1 for negatively curved, and 0 for flat. The scale factor $a$ parameterizes the expansion and curvature of space; it is related to the Hubble parameter $H$ by $H=\dot{a} / a$. One conventionally takes the 'big bang' itself to occur at $a=0$ and the present to be at $a=1 .^{11}$

A flat FRW spacetime has $k=0$. Only a specific value of the energy density $\rho$ will result in a universe with exactly flat spatial curvature. This is the critical density $\rho_{c r}$. It is obtained from the first Friedman equation by setting $k$ to zero:

$$
\rho_{c r}=\frac{3}{8 \pi} H^{2} .
$$

The present Hubble parameter $H_{0}$ and the present density $\rho_{0}$ may be determined from observations, thus allowing the comparison of $\rho_{0}$ and $\rho_{c r}$. These have been determined to be extremely close in recent years, although their approximate equality has been conspicuous for many decades. Thus, if our modeling assumptions are correct, we appear to live in a (very nearly) spatially flat universe.

It is straightforward to demonstrate the dynamical instability that features in the flatness problem, One way is to modify the first Friedman equation slightly, dividing it by the critical density $\rho_{c r}$ and defining a new parameter, the density parameter $\Omega=\rho / \rho_{c r}$. Then one has

${ }^{11}$ Of course the singularity is not a point of spacetime and did not occur according to the general theory of relativity, so $a=0$ is strictly not a valid parameter value of the scale factor, but any $a>0$ is. 


$$
1-\Omega=-\frac{k}{(a H)^{2}} .
$$

As we would like to see what happens when there are small departures from flatness and we do not care whether the departures are positive or negative, we may for convenience take the absolute value of both sides and ignore the $k=0$ case. We then have the following equation:

$$
|1-\Omega|=\frac{1}{(a H)^{2}} .
$$

So long as the universe is expanding, the right hand side is always increasing in time. Its time derivative is

$$
\frac{\mathrm{d}}{\mathrm{d} t} \frac{1}{(a H)^{2}}=-\frac{2 \ddot{a}}{\dot{a}^{3}} .
$$

If the universe only has normal matter in it, then $\ddot{a}$ is always negative (normal matter gravitates and hence decelerates expansion). Therefore we may conclude that $|1-\Omega|$ increases in time under small perturbations from flatness, and increasingly so. ${ }^{12}$

The instability of flat FRW spacetimes allows a fine-tuning argument to be made based on it. ${ }^{13}$ Our universe is presently observed to be expanding with a nearly flat spatial geometry. If it is exactly flat, then its initial conditions in the very early universe were such that it had exactly the critical density. If, however, it had ever so slightly different initial conditions, such that it had slightly less or slightly more than the critical density, then it would be nowhere near spatially flat today: it would be highly curved, in most cases to a degree that would not permit our existence. In other words, only initial conditions in a very narrow range would result in the presently observed universe. The big bang model, in short, requires that our universe be highly fine-tuned. One can do various calculations to get a sense of the degree of fine-tuning; Baumann $(2009,23)$, for example, calculates the fine-tuning to be one part in $10^{55}$ for initial conditions placed at the GUT scale.

There are various reactions one might have to the fact of this fine-tuning. One is, "so what?" If we trust our models and observations, then it is simply a logical consequence that the universe had to have had such-and-such initial conditions, within a range suggested by the uncertainty in observations. What does it matter that they could not have been much different? After all, presumably any physical system requires some particular initial conditions. Such an attitude appears to be partly behind the analysis of Earman and Mosterín (1999, 19-20), and it is presumably the reaction that many other philosophers of science, especially strongly empiricist ones, would take.

\footnotetext{
${ }^{12}$ For positively curved FRW spacetimes this is true only up to a point. These universes reach a maximum curvature, after which they contract into a 'big crunch'.

${ }^{13}$ Fine-tuning arguments take different forms and invoke varying considerations, as the general notion of fine-tuning arises in other contexts besides relativistic cosmology, for example high energy physics (Williams, 2015) and the notorious 'fine-tuning for life' problem debated by scientists, philosophers, and the religious. See (Friederich, 2018) for an introduction to these latter kinds. Dicke apparently was responsible for popularizing the flatness problem among physicists. See, e.g. (Dicke and Peebles, 1979).
} 
This is not the attitude that theoretical cosmologists take. They say that this finetuning indicates that the initial conditions required by the hot big bang model are special and therefore problematic. Unfortunately, they are not so clear about what exactly makes fine-tuned initial conditions problematic (McCoy, 2015). Cosmologists usually interpret this specialness in terms of likelihoods or probabilities. However, there are serious problems, both conceptual and technical, with interpreting fine-tuning problems in cosmology in this way (McCoy, 2018a). If cosmologists are merely reporting on their subjective degrees of belief, then it is unclear why anyone else should take their pronouncements very seriously. There should be some objective justification of likelihood attributions. Since likelihoods or probabilities are not part of the theory of general relativity, though, it is quite difficult to see from where they might come. ${ }^{14}$

Recalling the earlier episode's focus on stability, perhaps the specialness of finetuned initial conditions in the flatness problem is owed simply to the instability of the dynamics for the condition of flatness. Then, at least, it is justified to say that flatness is special, for under the FRW dynamics it is indeed unstable (and indeed, if this were the problem, then inflationary theory does solve the problem, by reversing the instability of flatness (McCoy, 2015)). The difficulty in this case, however, is the same as that raised before: namely, sustaining the claim that this instability is problematic. Again, cosmologists in the 1980s and afterwards generally do not seem to take the instability of flatness to be a problem as such however; rather the instability of flatness coupled with the observed approximate flatness of the universe requires special initial conditions, which they take to be problematic. Thus the reaction to instability is somewhat different in this episode compared to the previous one. Nevertheless, we see that in both cases instability is taken to have important consequences for theorizing, although what consequences those are vary, especially depending on whether instability is seen as a vice or a virtue. Moreover, some of the reactions, it seems, may be based on dubious philosophical assumptions, such as the aforementioned 'dogma of stability' (Schmidt, 2011) or the improbability of special initial conditions (Callender, 2004).

\section{Stability and Fragility}

While there are a variety of threads that one could pull from these two episodes, leading in many different directions, in this final section I want to keep a hold on the notion of stability and follow it into a more general context, particularly as it appears in dynamical systems theory. The precise notion of stability in dynamical systems theory is usually attributed to Andronov and Pontryagin, in their article "Grubye sistemy" (Coarse systems) (Andronov and Pontryagin, 1937), although this

\footnotetext{
${ }^{14}$ Interestingly, several physicists have made likelihood-based arguments that there is actually no flatness problem (Gibbons et al, 1987; Hawking and Page, 1988; Coule, 1995; Gibbons and Turok, 2008; Carroll and Tam, 2010). Their arguments, however, suffer from problems more or less as serious as those of the flatness problem supporters (Schiffrin and Wald, 2012; McCoy, 2017).
} 
was preceded by important work by Poincaré and Birkhoff, among others. ${ }^{15}$ Around the same time as this latter pioneering early work was being done on dynamical systems theory, the epistemic significance of stability was emphasized by Duhem (1962, Part 2, Ch. 3) and Poincaré (1952, I.IV.II) — even in the face of a growing appreciation of the mathematical significance of instability in dynamical systems.

I will make use of the narrative given by (Tavakol, 1991) to situate some of the main philosophical considerations. Tavakol argues that scientists generally assume, usually implicitly, that both real systems and mathematical models of those systems are structurally stable, that is, adopt the dogma of stability. He characterizes this assumption as follows (Tavakol, 1991, 148):

(a) Real systems are structurally stable in the sense that they do not change their qualitative behavior under small general perturbations.

(b) For mathematical models to be viable as models of real systems they similarly need to be structurally stable.

So, for example, in the context of cosmology this amounts to assuming that the universe does not change its qualitative behavior under small general perturbations and that our mathematical models should respect this. Since the Einstein static universe certainly does change its qualitative behavior under even a small scalar perturbation, changing from a static to a non-static universe, it violates the assumption. Similarly, since the Einstein-de Sitter universe changes from statically flat spatial curvature to dynamically changing spatial curvature, it too is not in accord with the assumption.

Tavakol offers two important reasons for adopting these assumptions, which he calls the 'stability framework'. One is that the framework addresses the problem of relating idealized models to real systems. The other is that the framework addresses the inaccuracy of observations.

First, idealization. There are a number of reasons that scientists use idealized models. Some are practical. For example, one must invoke simplifying assumptions to make solving actual problems tractable (particularly if the dynamics is nonlinear). Some have a more epistemic character. As described in section one, in the case where we use a simplified model to stand in for a real system, we would like to know that any differences between the model and the system will not undermine the model's predictions, explanations, etc. For example, our universe is clearly not exactly spatially homogeneous and isotropic, but we model it as if it were; if the universe were structurally stable, then its small departures from homogeneity and isotropy would not change its qualitative behavior of being (nearly) uniform. Our cosmological models should reflect that stability when it exists, and we can check that they do, in the example by introducing general perturbations into the FRW models to see if there are qualitative changes in behavior in the perturbed FRW models.

Second, inaccuracy. Real observations always involve some degree of inaccuracy due to error. The stability framework addresses this problem by insuring that small inaccuracies in measurement will not undermine the application of models whose

\footnotetext{
${ }^{15}$ For historical accounts of the development of dynamical systems, see, e.g., (Aubin and DahanDalmedico, 2002; Holmes, 2010).
} 
parameters depend on these measurement results. So long as the model accurately representing the real world is nearby ('a perturbation away'), then we can expect that predictions based on using the 'inaccurate' model will be approximately correct. Thus, as Tavakol says, the stability framework "facilitate[s] the task of interpreting observational and experimental data, by providing a theoretical framework...within which such data can be analysed" (Tavakol, 1991, 148).

Nevertheless, assumption (a) is not an insubstantial assumption, and it has plausibly been adopted largely for pragmatic reasons. As mentioned previously, that this is so has become especially clear in the past century thanks to the phenomenon of chaos in nonlinear dynamical systems, the applicability of which to real systems demonstrates that they are not necessarily structurally stable. Chaotic systems, characterized by a sensitive dependence on initial conditions and a mixing dynamics, entail a failure of deterministic predictability in chaotic models representing them (Batterman, 1993; Holt and Holt, 1993; Leiber, 1997; Werndl, 2009). This is yet another example of a violation of the stability framework. Thus this framework cannot be used in such cases to account for idealizations and measurement inaccuracy; indeed, chaotic systems present a significant challenge for the interpretation of empirical data and accounting for the confirmation of chaotic models (Rueger and Sharp, 1996; Koperski, 1998; Batitsky and Domotor, 2007), for one must appeal to alternative methods and justifications to secure the epistemic goods that were previously licensed by the stability framework.

If we must give up the stability framework, then Tavakol claims that there are two options: (1) hold onto dynamical determinism but adopt what he calls the 'fragility framework' or (2) maintain stability at the expense of determinism by introducing stochasticity. Each of these raises interesting philosophical challenges. On the one hand, adopting the fragility framework amounts to accepting that real systems may be structurally unstable, as a consequence of which our models must allow for the corresponding kinds of instabilities. Of course stability is not an all or nothing affair: one expects a spectrum of possibilities, in principle, between highly stable and highly fragile. This in mind, Kamminga and Tavakol (1993) suggest, for example, interpreting ceteris paribus clauses in laws of nature as identifying, roughly, the absence of relevant variability, which is to say the absence of the kind of perturbations which would lead to changes in qualitative behavior. Still, giving up the stability framework requires a different approach to understanding our modeling and testing practices, as just noted. On the other hand, Tavakol's example of maintaining stability involves a redefinition of stability, one which then involves the necessary introduction of an element of stochasticity. Stochasticity, of course, is a natural alternative to conventional stability, as Poincaré (1952, I.IV.II) and others pointed out long ago. If this stochasticity is to be objective, however, then there is a philosophical challenge to taking up this strategy, namely of grounding the objectivity of these deterministic probabilities (Lyon, 2011; Myrvold, 2012; McCoy, 2018b).

Applying these ideas in cosmology, then, either we accept the fragility framework in cosmological modeling (and its attendant philosophical challenges) or we adopt a statistical approach in cosmology (and its attendant philosophical chal- 
lenges). ${ }^{16}$ We are forced into this dilemma not just because many of the cosmological models that we know are unstable, however, but because we should expect instability in general in cosmology: it is a general feature of nonlinear dynamical systems, of which the systems described by general relativity are a kind. ${ }^{17}$ If this argument is right, then the rejection of a cosmological model, like the Einstein static universe, solely on the basis of its instability under certain perturbations is surely epistemically unjustified. It is, at best, a pragmatic decision which one might make in the hopes of discovering a stable model, or, at least, a model stable under perturbations deemed relevant for assessing whether the idealizations of the model are acceptable.

\section{Conclusion}

Determinism, probability, and causality are concepts that have long played important roles in scientific, physical, and cosmological thinking; they have also been central to philosophical discussions. The aim of this paper has been to draw some attention to the significant role that stability considerations too have played in cosmology. My focus has been on two cases, the Einstein static universe and the flatness problem, that I believe exhibit interesting aspects of stability in this historical thread. It would be a mistake, of course, to drive any strong, general conclusions on the basis of just these cases. Instead, I hope to have shown enough to suggest the significance of stability in cosmology, some of the roles it can play, and how attitudes towards it have changed.

In the first section I introduced the Einstein static universe and the de Sitter universe and derived some important consequences, such as the spherical geometry of space and the condition for stability of the former. I then showed how Eddington demonstrated the instability of the Einstein static universe to scalar perturbations of the energy density $\rho$ and pressure $p$. I related some of the historical consequences of Eddington's demonstration, including Einstein's disavowal of his model and the search by many cosmologists for a physical mechanism which could introduce a perturbation that would lead to an expanding universe, as observations suggested our universe was. Although empirical evidence provided the strongest argument for rejecting the Einstein static universe, it seems that Eddington's argument played an important role-certainly in the case of Einstein.

In the second section I explained the flatness problem, which arises in the context of the hot big bang model. Observations suggest that our universe is approximately spatially flat, but the spatially flat FRW spacetime is unstable under perturbations. This led some cosmologists to press a fine-tuning argument against the model, concluding that the special initial conditions required to account for flatness were prob-

\footnotetext{
${ }^{16}$ See (Tavakol and Ellis, 1988, 1990; Coley and Tavakol, 1992; Lidsey and Tavakol, 1993) for Tavakol's own application of his arguments to cosmology, including several examples.

17 This does not necessarily mean that we should expect cosmology to be chaotic, although that is a conceptual possibility that has been investigated. See, e.g. (Coleman and Pietronero, 1992).
} 
lematic in a way that required a theoretical modification of the big bang model. The proposed solution to the flatness problem was inflation, a stage of accelerated expansion in the very early universe. Although instability was not singled out as the problem of the flat FRW spacetime, it is only because of the particular kind of instability that the initial conditions are as special as they are, whether that specialness is understood in terms of probability or otherwise. The inflationary solution reverses the dynamics, so to speak, in such a way that flatness becomes stable and the initial conditions become less special.

In the third section I contextualized these two episodes by discussing stability in general, with special attention given to the advances in dynamical systems theory, and especially in nonlinear dynamics and chaos theory. I made use of Tavakol's alternative frameworks, the stability framework and fragility framework, discussing the epistemic issues associated with each one. Tavakol draws particular attention on the consequences of stability and fragility for idealization and inaccuracy. One important conclusion that can be drawn from this general context is that there are only pragmatic reasons to work within the stability framework, for it has become clear that physical systems do in fact exhibit instabilities of various kinds.

Reflecting back on the cosmological episodes, we can see that an outright rejection of the Einstein static universe is epistemically unjustified, although it perhaps can be motivated on pragmatic grounds. The response of Eddington, Lemaître, and others, to make this instability an opportunity, appears to be better motivated; in light of the subsequent fine-tuning arguments against the big bang model, however, we can easily imagine their proposal being subject to fine-tuning arguments of various kinds, for example in the posited equilibrium state, in the required perturbation to obtain an expanding universe of the appropriate kind, and so on. This supposition might make fine-tuning arguments appear spurious, and indeed this is a(n especially philosophical) reaction to the later flatness problem. Although characterizing what is problematic about special conditions in terms of instability or improbability cannot be sustained, as I have argued elsewhere (McCoy, 2018a), I also believe the empirical successes of inflationary cosmology should not be overlooked. The theory did solve conceptual problems which did lead to progress (McCoy, forthcoming). It is just not clear how to characterize these problems conceptually. One suggestion of

how that comes out of the discussion of stability here is that the instability of flat FRW spacetimes points to the specialness of the idealizations that lead to it. That, naturally, will have to be the topic for another time however.

\section{References}

Abraham, Ralph and Jerrold Marsden. 1978. Foundations of Mechanics, 2nd edn. Redwood City: Addison Wesley.

Andronov, Aleksandr and Lev Pontryagin. 1937. Grubye sistemy. Doklady Akademii Nauk SSSR 14:247-251. 
Aubin, David and Amy Dahan-Dalmedico. 2002. Writing the history of dynamical systems and chaos: Longue Durée and revolution, disciplines and cultures. Historia Mathematica 29(3):273-339.

Batitsky, Vidam and Zoltan Domotor. 2007. When good theories make bad predictions. Synthese 157(1):79-103.

Batterman, Robert. 1993. Defining chaos. Philosophy of Science 60(1):43-66.

Baumann, Daniel. 2009. TASI lectures on inflation. Available via arxiv.org. http://arxiv.org/abs/0907.5424. Cited 31 July 2018.

Callender, Craig. 2004. Measures, explanations and the past: Should 'special' initial conditions be explained? The British Journal for the Philosophy of Science 55:195-217.

Carroll, Sean and Haywood Tam. 2010. Unitary evolution and cosmological finetuning. Available via arxiv.org. http://arxiv.org/abs/1007.1417. Cited 31 July 2018.

Coleman, Paul and Luciano Pietronero. 1992. The fractal structure of the universe. Physics Reports 213:311-389.

Coley, Alan and Reza Tavakol. 1992. Fragility in cosmology. General Relativity and Gravitation 24(8):835-855.

Coule, David. 1995. Canonical measure and the flatness of a FRW universe. Classical and Quantum Gravity 12:455-469.

de Sitter, Willem. 1917a. On Einstein's theory of gravitation and its astronomical consequences, iii. Monthly Notices of the Royal Astronomical Society 78:3-28.

de Sitter, Willem. 1917b. Over de kromming der ruimte. Koninklijke Akademie van Wetenschappen te Amsterdam. Wis- en Natuurkundige Afdeeling Verslagen van de Gewone Vergaderingen 26:222-236.

de Sitter, Willem. 1917c. Over de relativiteit der traagheid: Beschouwingen naar aanleiding van Einsteins laatste hypothese. Koninklijke Akademie van Wetenschappen te Amsterdam Wis- en Natuurkundige Afdeeling Verslagen van de Gewone Vergaderingen 25:1268-1276.

Dicke, Robert and Jim Peebles. 1979. The big bang cosmology—enigmas and nostrums. In General Relativity: An Einstein Centenary Survey, eds. Stephen Hawking and Werner Israel, 504-517, Cambridge: Cambridge University Press.

Dingle, Herbert. 1933. On isotropic models of the universe, with special reference to the stability of the homogeneous and static states. Monthly Notices of the Royal Astronomical Society 94:134-158.

Duhem, Pierre. 1962. The Aim and Structure of Physical Theory. New York: Atheneum.

Earman, John. 2001. Lambda: The constant that refuses to die. Archive for History of Exact Sciences 55:189-220.

Earman, John and Jesus Mosterín. 1999. A critical look at inflationary cosmology. Philosophy of Science 66:1-49.

Eddington, Arthur. 1930. On the instability of einstein's spherical world. Monthly Notices of the Royal Astronomical Society 90:668-678. 
Einstein, Albert. 1917. Kosmologische betrachtungen zur allgemeinen relativitätstheorie. Sitzungsberichte der Königlich Preussischen Akademie der Wissenschaften (Berlin): 142-152

Einstein, Albert. 1923. Cosmological considerations on the general theory of relativity. In The Principle of Relativity, eds. Wilfrid Perrett and George Jeffrey, 177-188, London: Methuen and Co.

Einstein, Albert. 1931. Zum kosmologische problem der allgemeinen relativitätstheorie. Sitzungsberichte der Königlich Preussischen Akademie der Wissenschaften (Berlin): 235-237.

Einstein, Albert and Willem de Sitter. 1932. On the relation between the expansion and the mean density of the universe. Proceedings of the National Academy of Sciences 18:213-214.

Friederich, Simon. 2018. Fine-tuning. In The Stanford Encyclopedia of Philosophy, Spring 2018, ed. Edward Zalta. https://plato.stanford.edu/archives/spr2018/entries/fine-tuning/. Cited 31 July 2018.

Friedman, Aleksandr. 1922. Über die krümmung des raumes. Zeitschrift für Physik 10(1):377-386.

Friedman, Aleksandr. 1924. Über die möglichkeit einer welt mit konstanter negativer krümmung des raumes. Zeitschrift für Physik 21(1):326-332.

Gibbons, Gary and Neil Turok. 2008. Measure problem in cosmology. Physical Review D 77(063516).

Gibbons ,Gary, Stephen Hawking, and John Stewart. 1987. A natural measure on the set of all universes. Nuclear Physics B 281:736-751.

Hawking, Stephen and George Ellis. 1973. The Large Scale Structure of SpaceTime. Cambridge: Cambridge University Press.

Hawking, Stewart and Don Page. 1988. How probable is inflation? Nuclear Physics B 298:789-809.

Holmes, Philip. 2010. A short history of dynamical systems theory: 1885-2007. In History of Mathematics, eds. Vagn Hansen and Jeremy Gray, 115-138, Singapore: Eolss.

Holt, Lynn and Glynn Holt. 1993. Regularity in nonlinear dynamical systems. The British Journal for the Philosophy of Science 44(4):711-727.

Hubble E (1929) A relation between distance and radial velocity among the extragalactic nebulae. Proceedings of the National Academy of Sciences 15:168-173

Kamminga, Harmke and Reza Tavakol. 1993. How untidy is god's mind? a note on the dynamical implications of Nancy Cartwright's metaphysics. The British Journal for the Philosophy of Science 44(3):549-553.

Koperski, Jeffrey. 1998. Models, confirmation, and chaos. Philosophy of Science 65(4):624-648.

Kragh, Helge. 2007. Conceptions of the Cosmos. Oxford: Oxford University Press.

Leiber, Theodor. 1997. On the actual impact of deterministic chaos. Synthese 113(3):357-379. 
Lemaître, Georges. 1927. Un univers homogène de mass constant et de rayon croissant, rendant compte de la vitesse radiale des nébuleuses extra-galactiques. Annales de la Société Scientifique de Bruxelles Série A 47:49-59.

Lemaitre, Georges. 1931. The expanding universe. Monthly Notices of the Royal Astronomical Society 91:490-501.

Lidsey, James and Reza Tavakol. 1993. On the correspondence between theory and observations in inflationary cosmology. Physics Letters B 309(1-2):23-27.

Lyon, Aiden. 2011. Deterministic probability: neither chance nor credence. Synthese 182:413-432.

Malament, David. 2012. Topics in the Foundations of General Relativity and Newtonian Gravity Theory. Chicago: University of Chicago Press.

McCabe, Gordon. 2004. The structure and interpretation of cosmology: Part igeneral relativistic cosmology. Studies in History and Philosophy of Modern Physics 35:549-595.

McCoy, Casey. 2015. Does inflation solve the hot big bang model's fine-tuning problems? Studies in History and Philosophy of Modern Physics 51:23-36.

McCoy, Casey. 2016. Philosophical implications of inflationary cosmology. PhD thesis

McCoy, Casey. 2017. Can typicality arguments dissolve cosmology's flatness problem? Philosophy of Science 84(5):1239-1252.

McCoy, Casey. 2018a. The implementation, interpretation, and justification of likelihoods in cosmology. Studies in History and Philosophy of Modern Physics 62:19-35.

McCoy, Casey. 2018b. No chances in a deterministic world. Available via philsciarchive.pitt.edu. http://philsci-archive.pitt.edu/13166/. Cited 31 July 2018.

McCoy, Casey. Forthcoming. Epistemic justification and methodological luck in inflationary cosmology. The British Journal for the Philosophy of Science 0:1-21.

McCrea, William and George McVittie. 1930. On the contraction of the universe. Monthly Notices of the Royal Astronomical Society 91:128-133.

McCrea, William and George McVittie. 1931. The expanding universe. Monthly Notices of the Royal Astronomical Society 92:7-12.

McVittie, George. 1931. The problem of $\mathrm{n}$ bodies and the expansion of the universe. Monthly Notices of the Royal Astronomical Society 91:274-283.

Myrvold, Wayne. 2012. Deterministic laws and epistemic chances. In Probability in Physics, eds. Yemima Ben-Menahem and Meir Hemmo, 73-85, Berlin: Springer.

Norton, John. 1992. A paradox in newtonian gravitation theory. PSA: Proceedings of the Biennial Meeting of the Philosophy of Science Association 1992:412-420.

Nussbaumer, Harry. 2014. Einstein's conversion from his static to an expanding universe. European Physical Journal H 39:37-62.

O'Raifeartaigh, Cormac and Brendan McCann. 2014. Einstein's cosmic model of 1931 revisited: an analysis and translation of a forgotten model of the universe. European Physical Journal H 39(1):63-85.

O'Raifeartaigh, Cormac, Michael O'Keeffe, Werner Nahm, and Simon Mitton. 2017. Einstein's 1917 static model of the universe: a centennial review. European Physical Journal H 42(3):431-474. 
Poincaré, Henri. 1952. Science and Method. New York: Dover.

Robertson, Howard. 1933. Relativistic cosmology. Reviews of Modern Physics 5(1):62-90.

Rueger, Alexander and David Sharp. 1996. Simple theories of a messy world: Truth and explanatory power in nonlinear dynamics. The British Journal for the Philosophy of Science 47(1):93-112.

Schiffrin, Joshua and Robert Wald. 2012. Measure and probability in cosmology. Physical Review D 86(023521).

Schmidt, Jan. 2011. Challenged by instability and complexity. In Philosophy of Complex Systems, ed. Cliff Hooker, 223-254, Oxford: Elsevier.

Sen, Nikhil. 1935a. On a minimum property of the Friedmann space. Zeitschrift für Astrophysik 9:315-318.

Sen, Nikhil. 1935b. On the stability of cosmological models with nonvanishing pressure. Zeitschrift für Astrophysik 10:291-296.

Slipher, Vesto. 1912. The radial velocity of the andromeda nebula. Lowell Observatory Bulletin 2(8):56-57.

Slipher, Vesto. 1915. Spectrographic observations of nebulae. Popular Astronomy 23:21-24.

Slipher, Vesto. 1917. Nebulæ. Proceedings of the American Philosophical Society 56(5):403-409.

Slipher, Vesto. 1921. Two nebulae with unparalleled velocities. Popular Astronomy 29:128.

Smeenk, Chris. 2014. Einstein's role in the creation of relativistic cosmology. In: The Cambridge Companion to Einstein, eds. Michel Janssen and Christoph Lehner, 228-269, Cambridge: Cambridge University Press.

Tavakol, Reza. 1991. Fragility and deterministic modelling in the exact sciences. The British Journal for the Philosophy of Science 42:147-156.

Tavakol, Reza and George Ellis. 1988. On the question of cosmological modelling. Physics Letters A 130:217-224.

Tavakol, Reza and George Ellis. 1990. Stability of cosmological models. Physics Letters A 143(1-2):8-12.

Tolman, Richard. 1934. Relativity, Thermodynamics and Cosmology. Oxford: Clarendon Press

Wald, Robert. 1984. General Relativity. Chicago: University of Chicago Press.

Werndl, Charlotte. 2009. What are the new implications of chaos for unpredictability? The British Journal for the Philosophy of Science 60(1):195-220.

Williams, Porter. 2015. Naturalness, the autonomy of scales, and the 125gev Higgs. Studies in History and Philosophy of Modern Physics 51:82-96. 